\title{
Size Constancy at Birth: Newborn Infants' Responses to Retinal and Real Size
}

\author{
Alan Slater, Anne Mattock, and Elizabeth Brown \\ Washington Singer Laboratories, University of Exeter
}

\begin{abstract}
Two experiments are described whose aim was to investigate whether perception of size at birth is determined solely by proximal (retinal) stimulation, or whether newborn babies have the ability to perceive an object's real size across changes in distance. In Experiment 1, preferential looking between pairs of stimuli which varied in real size and viewing distance was found to be solely determined hy retinal size, suggesting that changes to proximal stimulation can have profound effects on newborns' looking behavior. However, in Experiment 2 newborns were desensitized to changes in distance (and retinal size) during familiarization trials, and subsequently strongly preferred a different sized object to the familiar one, suggesting that the real size had been perceived as constant across the familiarization trials. These results confirm Granrud's (1987) findings that size constancy is present at birth. (c) 1990 Academic Press, Inc.
\end{abstract}

One organizing feature of perception which contributes to the perceived stability of the visual world is size constancy, that is, seeing an object as its real size despite changes that occur to the size of its retinal image as its viewing distance from an observer varies. It seems certain that size constancy is present at some point in infants' visual perception, but exactly when, and whether learning and experience are required for its development, are not fully known. Evidence for size constancy in early infancy was reported by Bower $(1964,1966)$. Eight-week-old infants were conditioned to give head turns when a $30-\mathrm{cm}$ cube was presented $1 \mathrm{~m}$ away; and they subsequently gave more conditioned responses to the same-size cube at a distance of $3 \mathrm{~m}$, whose retinal image was onethird the size of the training stimulus, than to a $90-\mathrm{cm}$ cube at $3 \mathrm{~m}$, whose retinal image size was the same as the training stimulus. The data

This research was supported by Grant RC 00232466 to the first author from the Economic and Social Research Council. We are indebted to the staff of the Maternity Ward, Royal Devon and Exeter Hospital, Exeter, England, and to all the subjects' mothers for their cooperation and assistance. We thank Catriona Ryan who helped in the data collection. We also thank the reviewers for their helpful comments on an earlier version of the manuscript. Reprint requests should be addressed to Alan Slater, Department of Psychology, Washington Singer Laboratories, University of Exeter, Exeter EX4 4QG, UK. 
from more recent studies (Day \& McKenzie, 1981; McKenzie, Tootell, $\&$ Day, 1980), using recovery from habituation as an index of size constancy, confirmed that the ability to perceive the true size of an object is present by 18 weeks: in these experiments infants who had been habituated to one object subsequently dishabituated to an object that was a different real size, but did not dishabituate to an object that was the same size but at a different distance.

These findings suggest that size constancy is present in early infancy, and a recent study by Granrud (1987) suggests that it may be present at birth. In Granrud's study, newborn infants were tested in either a "Constant-Size" or "Variable-Size" condition. In the Constant-Size conditions the infants were shown a 6-cm diameter sphere at three distances: 16,24 , and $32 \mathrm{~cm}$. The Variable-Size group were shown (separately) three different-sized spheres, 3,6, and $12 \mathrm{~cm}$ in diameter presented, respectively, at distances of 16,24 , and $32 \mathrm{~cm}$. The spheres subtended identical visual angles in the two conditions, and there were two 20-s trials for each stimulus, giving six trials for each group. The ConstantSize group showed a significant decrease in fixation time from Trial 1 to Trial 6 but the Variable-Size group did not, suggesting that the former group was seeing a same-sized sphere over trials, and the latter group perceived that different-sized spheres were presented over trials. These results indicate that newborn infants possess some degree of size constancy. An excellent and thorough recent review of visual size constancy in infancy is provided by Day (1987).

The experiments described here were intended to look for the presence of size constancy at birth. Experiment 1 used preferential looking procedures to see in what way size preferences at birth are influenced by retinal and/or real size. In Experiment 2, a familiarization/recovery-tonovelty procedure was used to see if newborns can perceive an object's constant real size across changes in distance (and hence, changes in retinal size). The experiments are presented after description of the general method.

\section{METHOD}

Subjects

Subjects were selected from the maternity ward of the Royal Devon and Exeter Hospital, Heavitree, Devon, and were tested after their midday feed. Throughout testing they were in the behavioral state of alert inactivity (Ashton, 1973). Forty-eight babies were tested in the first experiment, 23 males and 25 females, mean age- 2 days $17 \mathrm{~h}$; range $9 \mathrm{~h}$ to 7 days $22 \mathrm{~h}$; and 12 in the second experiment, 7 males and 5 females, mean age- 2 days $7 \mathrm{~h}$; range $13 \mathrm{~h}$ to 5 days $0 \mathrm{~h}$. A further 21 babies were seen but could not be included as subjects because of fussing, crying, 
or sleeping $(N=9)$, or position bias on paired-stimulus preferential looking trials or on postfamiliarization trials $(N=12)$.

\section{Stimuli}

The stimuli were white cubes with black edging, and can be seen in Figs. 1 and 2. Two sizes of cube were used: the smaller had 5.1-cm sides and the larger had $10.2-\mathrm{cm}$ sides. During presentation three faces of the cube(s) were visible to the infants (see Figs. 1 and 2) and they were shown at different viewing distances (described below), calculated as the distance from the subject's eyes to the nearest point of the cube(s). At the closest viewing distance of $23 \mathrm{~cm}$, the small and large cubes subtended maximum visual angles, respectively, of $18^{\circ}$ and $37^{\circ}$, and at the farthest distance of $69 \mathrm{~cm}$ the maximum visual angles of the small and large cubes, respectively, were $6^{\circ}$ and $12^{\circ}$.

\section{Apparatus}

The stimuli were presented against a background matt, gray-painted screen, and the sides of the viewing chamber were hung with gray curtain material (see Fig. 1). Illumination was provided by two striplights, one overhead and the other on the floor. The luminance levels of the white and black parts of the stimuli and of the gray background averaged, respectively, 70,20 , and $44 \mathrm{~cd} / \mathrm{m}^{-2}$. To present the stimuli, horizontal holder arms (either one or two, see below) were used which could be moved along the baby's line of sight to vary the viewing distance: these holder arms could not be seen by the babies and an adult viewing from the baby's eye view saw the cube(s) apparently suspended in space (see Fig. 2). Marks on the holder arms allowed speedy and accurate changes of viewing distance. A Rustrak Event Recorder was used in association with a millisecond timer to record the infants' fixations.

\section{Procedure}

Each subject was brought to the experimental room, on the maternity ward of the hospital, and seated upright on one experimenter's knee. Eye position was continuously monitored throughout, by means of pointers located on the floor and on the sides of the viewing chamber. Apart from occasional sudden movements by the subjects, which were easily corrected, viewing distance was maintained to an accuracy of $\pm 2 \mathrm{~cm}$. For paired stimulus presentation (the preference studies of Experiment 1 and the postfamiliarization test trials of Experiment 2), the midpoints of the two stimuli were separated by a visual angle of $32^{\circ}$ (i.e., the horizontal axes of the stimuli were positioned $16^{\circ}$ to either side of the straight-ahead position). For single presentation (the familiarization trials of Experiment 2), the stimulus was in the straight-ahead position.

Either one or two experienced observers recorded the infants' fixa- 
tions, from peepholes behind and to the left and/or right of the viewing chamber (see Fig. 1): at no time were these observers visible to the infants, and the observers had no expectations as to the outcome of the experiments. The testing procedure is illustrated in Fig. 1 which shows a baby presented with stimulus pairing (5) of Experiment 1, which is the same as the pairing presented on the test trials of Experiment 2. Figure 2 shows the infant's-eye-view of the stimuli in this pairing.

Typically in this sort of research the criterion of fixation is the reflection of the stimulus over the infant's cornea. In the experiments described here this was often not possible because of the luminance levels used, and the observers made moment-to-moment decisions as to whether the babies were looking at the single stimulus (the familiarization trials, Experiment 2) or at one or the other of the paired stimuli (Experiment 1 and the test trials of Experiment 2). In practice this is a highly reliable means of measuring newborn infants' fixations: for the paired-stimulus trials of 10 subjects, two independent observers recorded the infants' fixations on the stimuli. The interobserver agreement was high (Pearson $r=0.947)$. Other procedural details are given in the description of the experiments.

\section{EXPERIMENT 1}

Stimulus size is a dimension which affects preferential looking in newborn babies. Brennan, Ames, and Moore (1966), and Hershenson (1964) reported that newborns looked more at the largest stimulus shown: the stimuli were checkerboards or squares. However, Slater and Sykes (1977), whose stimuli were square-wave gratings, found that newborns preferred an intermediate range of spatial frequencies, rather than the

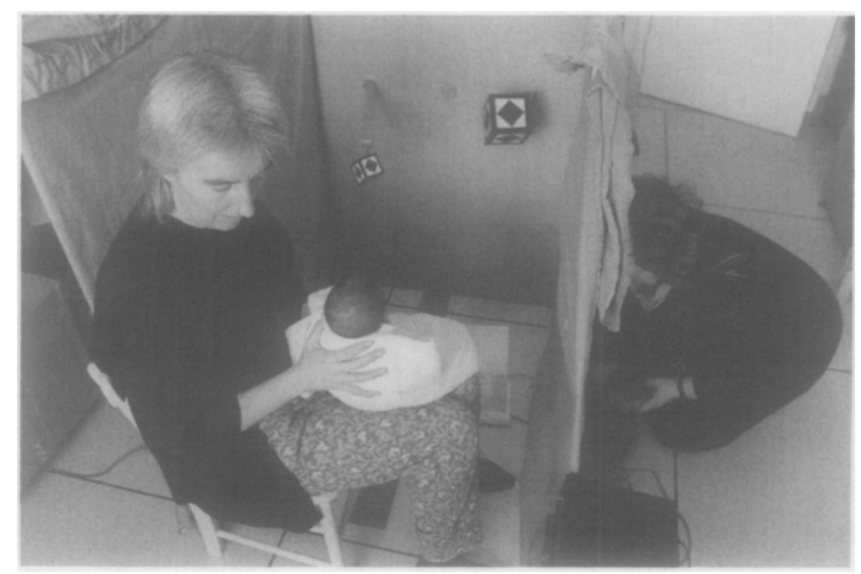

Fig. 1. Illustration of the testing procedure. 


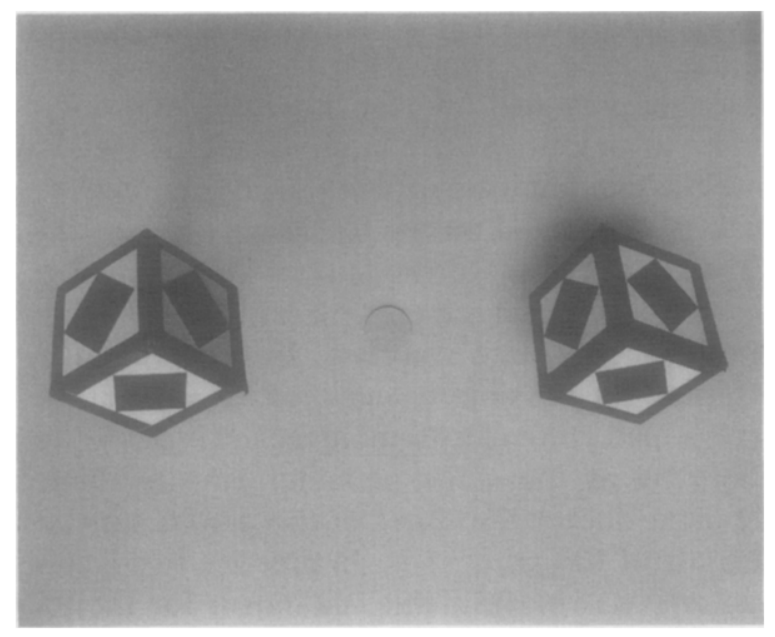

Fig. 2. The stimuli shown to the infants. This photograph, taken from the babies' viewing position, shows the small cube on the left at a distance of $30.5 \mathrm{~cm}$, and the large cube on the right at a distance of $61 \mathrm{~cm}$.

largest stripes shown; Slater and Morison (1985) found that newborns looked at a square in preference to a larger trapezium. Thus, it is clear that while size can be a powerful determinant of preferences, no easy predictions can be made as to what preferences might be found for stimuli that have not previously been shown to newborns, and we also do not know what effects viewing distance may have on preferences. The present experiment was designed to obtain this information: while it was expected that the variations in stimulus size and distance that were used would affect preferences, no more specific hypotheses were entertained.

\section{Method}

There were eight preferential looking stimulus pairings, and a minimum of eight subjects were tested in each. No subject was tested with more than three pairings. Each stimulus pairing was shown for two trials, each trial continuing until $20 \mathrm{~s}$ of looking had accumulated, and each stimulus changed its left/right position (while staying the same viewing distance) from trial 1 to trial 2 . Within a pairing, order of presentation was counterbalanced across subjects. The mean time to change the left/right stimulus positions was $10.5 \mathrm{~s}$, and the mean time to change from one stimulus pairing to the next was $22 \mathrm{~s}$.

The stimuli and distances in each pairing are identified in Table 1. These pairings were chosen because they allow quantification of changes in preferential looking that may result from systematic changes to size and distance. Pairings (7) and (8) were carried out after the others in 
TABLE 1

Experiment 1: Results of Preference Pairings

\begin{tabular}{crccc}
\hline Condition & $N$ & $\begin{array}{c}\text { Nonpreferred } \\
\text { cube and } \\
\text { viewing distance } \\
(\mathrm{cm})\end{array}$ & $\begin{array}{c}\text { Preferred } \\
\text { cube and } \\
\text { viewing distance } \\
(\mathrm{cm})\end{array}$ & $\begin{array}{c}\text { Mean percent } \\
\text { preference }\end{array}$ \\
\hline 1 & $8(8)$ & S, 30.5 & L, 30.5 & $92.8^{*}$ \\
2 & $8(8)$ & S, 61 & L, 61 & $89.7^{*}$ \\
3 & $8(8)$ & S, 61 & S, 30.5 & $87.7^{*}$ \\
4 & $8(8)$ & L, 61 & L, 30.5 & $89.1^{*}$ \\
5 & $16(8)$ & L, 61 & S, 30.5 & 50.6 \\
6 & $8(8)$ & S, 61 & L, 30.5 & $98.3^{*}$ \\
7 & $9(9)$ & L, 69 & S, 23 & $89.0^{*}$ \\
8 & $10(8)$ & S, 30.5 & L, 46 & $73.9^{*}$ \\
\hline
\end{tabular}

Note. $\mathrm{S}=$ Small cube; $\mathrm{L}=$ Large cube. Subject numbers in parentheses are those looking more at the preferred cube.

${ }^{*}$ Indicates significance beyond $p=.01$, smallest $t(7)=3.46$.

order to clarify problems of interpretation that were apparent from the results of preferences (1) to (6). More subjects were intentionally tested in (5) since it was intended at the outset that this pairing would be used in the test trials of Experiment 2. The rationale underlying the choice of preferences is clarified in the next section.

\section{Results and Discussion}

The results, expressed as the percentage of looking time spent viewing the preferred stimulus, are given in Table 1 . Seven of the pairings produced very strong, and highly significant, preferences, and the results from all eight pairings can be described by a simple rule: "look longest at the stimulus which gives the largest retinal image." Thus, for pairings (1) and (2), the largest cube was preferred to the smaller when both were at the same viewing distance. For pairings (3) and (4), the nearer of two same-sized cubes was preferred, and for pairing (6) the nearer, and larger, of two different-sized cubes was preferred. For pairing (7), the nearer, and smaller, and for pairing (8), the farther, and larger, of two differentsized cubes were preferred: for both of these pairings the preferred stimulus gave the largest retinal image. For pairing (5), where neither stimulus was preferred, the large cube was twice the distance of the small one and both gave the same retinal size.

These findings are a clear demonstration that retinal image size is a highly salient determinant of newborn preferential looking. Whether newborn babies can perceive an object as being the same real size, independently of its retinal size, is the focus of Experiment 2. 


\section{EXPERIMENT 2}

In this experiment each infant was given six familiarization trials with one stimulus (either the small or the large cube), this being a different distance on each trial. A similar procedure was used in shape constancy experiments by Caron, Caron, and Carlson (1979) with three-month-old infants, and by Slater and Morison (1985) with newborns. The intention of these trials was progressively to desensitize the infants to changes in retinal size in the hope that this would direct attention to the cube's real size. After this familiarization period the small and large cubes were shown at different distances so that their retinal sizes were the same, for two test trials, with the familiarized cube at a different distance (and hence with a different retinal size) from any shown before. This precaution was to ensure that the subjects could not be basing their responses on the test trials on any specific cues detected during the familiarization trials.

\section{Method}

Six newborns were familiarized to the small cube and six to the large. Those familiarized to the small cube were shown it at the following distances (in cm): (a) 23; (b) 53; (c) 38; (d) 69; (e) 46; and (f) 61. Those familiarized to the large cube were shown it at these distances (in $\mathrm{cm}$ ): (a) 23 ; (b) 46 ; (c) 30.5 ; (d) 69 ; (e) 53 ; and (f) 38 . The subjects in each of the familiarization conditions were presented the stimulus in a different order of distances, but with the sequence maintained across subjects: thus, the first subject in each condition was shown the stimulus in the order $a, b, c, d, e, f$; the second subject the order $b, c, d, e, f, a ; \ldots$; the sixth subject the order $f, a, b, c, d, e$. This order of stimulus presentation (rather than, say, a random order) was chosen in order to maximize distance changes across trials: the minimum shift in distance from one trial to the next was $15 \mathrm{~cm}$. In order to ensure that each subject had the same exposure to the familiarized stimulus at its different distances, each trial continued until $25 \mathrm{~s}$ of looking had accumulated, at which point the stimulus was moved to its next distance and the next trial began. The mean intertrial interval, defined as the period from the last look at the stimulus at one distance to the first look at the stimulus at the next, was $15.6 \mathrm{~s}$. During this interval, the experimenter who held the babies turned them away from the viewing area while the stimulus distance was changed (the change itself took a maximum of $2 \mathrm{~s}$ ), so that at no time did the infants see the stimulus in motion. When the familiarization trials ended two test trials were given, in which the small cube at $30.5 \mathrm{~cm}$ was paired with the large cube at $61 \mathrm{~cm}$ : this is the same as pairing (5) of Experiment 1 and the details of presentation are the same as described earlier. The mean interval between the familiarization and test trials was $18.6 \mathrm{~s}$. Several alternative same-sized cubes were available 
and as a precaution the "familiar," same-sized cube on the test trials was different from that shown on the familiarization trials.

\section{Results}

Each subject looked at the stimulus for the same amount of time (150 s) on the familiarization trials. To accumulate this looking time the subjects familiarized to the small cube took an average of $37 \mathrm{~s}$ per trial, while those familiarized to the large cube averaged $27.6 \mathrm{~s}$ : this difference, which is perhaps a further indication of the greater salience of the larger stimulus, was not significant.

The dependent variable of most interest is the time spent looking at the novel- and familiar-sized stimuli on the postfamiliarization test trials. These results are given for the individual subjects in Table 2. Every one of the subjects spent more time looking at the novel-sized stimulus on these trials, and the novelty preferences were surprisingly large: for those subjects familiarized to the small cube the novel, larger cube attracted a mean of $79.4 \%$ of the looking time, while for those familiarized to the large cube the novel, smaller cube attracted a mean of $88.3 \%$ of the looking time. The overall mean novelty preference was $83.8 \%$, which was highly significant $[t(11)=10.2, p<.0001]$, and it was significantly different from the preferences shown by those infants in pairing (5) of Experiment 1 who were presented the same stimulus pairing but without prior familiarization $[t(26)=3.32, p<.005$, one-tailed].

\section{GENERAL DISCUSSION}

The results from these experiments suggest that both retinal and real size are detected, and responded to, by newborns. The findings from the preference studies of Experiment 1 demonstrate unequivocally that newborn babies' preferential looking can be powerfully affected by changes to an object's retinal image size; indeed, in this experiment no variation in response attributable to objects' real sizes could be detected.

TABLE 2

Experiment 2: Novelty Preferences

\begin{tabular}{|c|c|c|c|}
\hline \multicolumn{2}{|c|}{ Familiarized to small cube } & \multicolumn{2}{|c|}{ Familiarized to large cube } \\
\hline Subject & $\begin{array}{c}\text { Novelty preference } \\
(\%)\end{array}$ & Subject & $\begin{array}{c}\text { Novelty preference } \\
(\%)\end{array}$ \\
\hline 1 & 72.5 & 7 & 92.5 \\
\hline 2 & 85 & 8 & 77.5 \\
\hline 3 & 83.8 & 9 & 88.8 \\
\hline 4 & 92.5 & 10 & 95.0 \\
\hline 5 & 88.8 & 11 & 85.0 \\
\hline 6 & 53.8 & 12 & 91.2 \\
\hline
\end{tabular}


However, the strong preferences for the novel-sized cube that were found on the test trials of Experiment 2 are equally clear in demonstrating that the newborn subjects were able to perceive the constant real size of the cube that was shown on the familiarization trials. These trials may have provided the experience required for the babies to detect the stimulus invariance (the real size) from the retinally-variable stimulus input. In this experiment, the novelty responses could not be based on perceived distance, since stimulus distance varied during the familiarization trials; neither could they be based on retinal size, since retinal size varied during familiarization, and the retinal sizes of the test stimuli were the same. These findings confirm and extend those of Granrud (1987): size constancy, therefore, would not seem to be dependent upon extensive learning and experience for its acquisition in infancy. Rather, like shape constancy, it is an organizing feature of perception that is present at or soon after birth.

\section{REFERENCES}

Ashton, R. (1973). The state variable in neonatal research: A review. Merrill-Palmer Quarterly, 19, 3-20.

Bower, T. G. R. (1964). Discrimination of depth in premotor infants. Psychonomic Science, $1,368$.

Bower, T. G. R. (1966). The visual world of infants. Scientific American, 215(6), 80-92.

Brennan, W. M., Ames, E. W., \& Moore, R. W. (1966). Age differences in infants' attention to patterns of different complexities. Science, 151, 354-356.

Caron, A. J., Caron, R. F., \& Carlson, V. R. (1979). Infant perception of the invariant shape of objects in slant. Child Development, 50, 715-721.

Day, R. H. (1987). Visual size constancy in infancy. In B. E. McKenzie \& R. H. Day (Eds.), Perceptual development in early infancy: Problems and issues. Hillsdale, NJ: Erlbaum.

Day, R. H., \& McKenzie, B. E. (1981). Infant perception of the invariant size of approaching and receding objects. Developmental Psychology, 17, 670-677.

Granrud, C. E. (1987). Size constancy in newborn human infants. Investigative Ophthalmology and Visual Science, 28, (Supplement), 5.

Hershenson, M. (1964). Visual discrimination in the human newborn. Journal of Comparative and Physiological Psychology, 58, 270-276.

McKenzie, B. E., Tootell, H. E., \& Day, R. H. (1980). Development of visual size constancy during the first year of human infancy. Developmental Psychology, 16, 163174.

Slater, A., \& Morison, V. (1985). Shape constancy and slant perception at birth. Perception, $14,337-344$.

Slater, A., \& Sykes, M. (1977). Newborn infants' visual responses to square wave gratings. Child Development, 48, 545-554.

Received: June 16, 1989; Revised: August 22, 1989. 